Revue de l'Institut des langues et cultures

d'Europe, Amérique, Afrique, Asie et Australie

$11 \mid 2009$

Langues \& cultures de spécialité à l'épreuve des médias

\title{
Discours de vulgarisation à travers différents médias ou les tribulations des termes scientifiques
}

Le cas de la médecine

Elodie Vargas

(2) OpenEdition

Journals

Édition électronique

URL : http://journals.openedition.org/ilcea/217

DOI : $10.4000 /$ ilcea. 217

ISSN : 2101-0609

Éditeur

UGA Éditions/Université Grenoble Alpes

Édition imprimée

ISBN : 978-2-84310-179-3

ISSN : 1639-6073

Référence électronique

Elodie Vargas, «Discours de vulgarisation à travers différents médias ou les tribulations des termes

scientifiques », ILCEA [En ligne], 11 | 2009, mis en ligne le 30 avril 2009, consulté le 08 mars 2021.

URL : http://journals.openedition.org/ilcea/217 ; DOI : https://doi.org/10.4000/ilcea.217

Ce document a été généré automatiquement le 8 mars 2021.

(c) ILCEA 


\section{Discours de vulgarisation à travers différents médias ou les tribulations des termes scientifiques}

Le cas de la médecine

\section{Elodie Vargas}

\section{Introduction}

1 La vulgarisation trouve sa raison d'être dans les problèmes de compréhension qu'éprouve le non-spécialiste lorsqu'il se heurte à un domaine de spécialité et à la langue qui en est son vecteur. Elle a pour but d'établir une communication entre communauté spécialiste et communauté "profane", faisant ainsi progresser la connaissance et la culture de cette dernière. Peut-on pour autant considérer qu'il n'existe qu'un type unique de vulgarisation ou doit-on au contraire parler de «modes de vulgarisation »? C'est à cette question que se propose de répondre cette contribution en mettant en regard les ouvrages de vulgarisation, les émissions de vulgarisation télévisées et les sites Internet spécialisés. La question à la base de notre exposé est ainsi de savoir si les termes et les « combinaisons lexicales spécialisées » (au sens de M.C. L'Homme) ${ }^{1}$ subissent le même traitement au niveau de la vulgarisation dans les différents médias présentés. Nos analyses reposent sur un corpus allemand se composant de 24 ouvrages de vulgarisation destinés aux enfants, adolescents, adultes profanes et adultes plus initiés, avec des thèmes communs à ces quatre publics, d'émissions télévisées de vulgarisation, et enfin de sites Internet spécialisés.

\section{Particularités du discours de vulgarisation dans les ouvrages et les magazines de vulgarisation}

2 L'adaptation au lecteur profane se réalise de diverses façons. Le rôle de l'illustration (dessins, croquis, images) est, bien sûr, un élément d'aide incontestable. Au niveau 
intertextuel, cette adaptation se marque par une simplification, résultant de la suppression d'informations intéressantes uniquement pour les spécialistes (noms des auteurs d'une découverte, informations théoriques sujettes à discussion, descriptions précises des objets analysés, etc.). Au niveau intratextuel, les opérations de reformulation constituent le maillon central des textes de vulgarisation. Des séquences contenant des termes ou expressions spécialisés et des séquences renfermant des équivalents ne relevant pas de discours spécialisés se succèdent, les unes à la suite des autres, se reformulant les unes les autres et assurant ainsi le passage entre monde des spécialistes et monde des profanes. Ces reformulations permettent un va-et-vient continuel entre termes ou combinaisons lexicales spécialisés et mots ou expressions du code courant (soit en dénomination, soit en définition/explication, ce qui constitue l'opération inverse.) Il convient donc de préciser ce que l'on entend dans ce cadre-là par « reformuler » et « reformulation ».

3 Nous avons défini la reformulation intratextuelle comme une opération discursive métalangagière ${ }^{2}$. Par cette opération, le locuteur unit de manière stratégique - et le temps d'un discours - deux énoncés ou segments d'énoncés. En effet, le locuteur, après un dit premier, revient sur celui-ci et ajoute un autre "dit» (que nous appelons « redit). Ce dernier doit être compris (grâce à divers signaux explicites) comme une formulation autre du premier. Ces deux segments sont identiques référentiellement. La reformulation est dite "paraphrastique ${ }^{3}$, si le redit (ou "segment reformulateur») renferme des informations allant dans le même sens que celles contenues dans le dit (ou «segment source») et proposant une image similaire du référent commun. La reformulation intratextuelle est donc définie au niveau informationnel et non sémantique. Toute reformulation intratextuelle peut être schématisée sous la forme $\mathrm{xRy}$ où $\mathrm{x}$ est le segment source, $\mathrm{y}$ le segment reformulateur et $\mathrm{R}$ la marque de reformulation, ainsi que le montre l'exemple (1) dans lequel « Leukozyten » constitue le segment source, le «dit», "weiße Blutkörperchen» constitue le segment reformulateur, le « re-dit » (en gras), les deux étant liés par la marque de reformulation « oder » (en gras souligné) :

(1) Leukozyten oder weiße Blutkörperchen sind farblose, kernhaltige Blutzellen. Sie haben keine feste Form. (Biologie, p. 185)

$\rightarrow$ Les leucocytes ou globules blancs sont des cellules sanguines incolores à noyau.

Ils n'ont pas de forme définie.

Dans la mesure où les deux segments renvoient au même référent, ceux-ci sont dans les cas de reformulations paraphrastiques permutables, ainsi que le montre (1a) :

(1a) Weiße Blutkörperchen oder Leukozyten sind farblose, kernhaltige Blutzellen. Sie haben keine feste Form.

$\rightarrow$ Les globules blancs ou leucocytes sont des cellules sanguines incolores à noyau. Ils n'ont pas de forme définie.

5 Cette permutation apporte bien sûr un changement du point de vue informationnel, les visées communicatives étant alors différentes.

6 Le segment reformulateur apporte un supplément d'informations, son statut est celui "d'un ajout, détaché, suppressible, doublant le terme de départ intégré à la construction de la phrase» (J. Authier-Revuz, 1982, 40). Non obligatoire, il est suppressible (voir $1 \mathrm{~b}$ ci-dessous), mais au niveau phrastique uniquement : en effet, au niveau textuel, le segment reformulateur est souvent indispensable, puisque certains enchaînements transphrastiques peuvent se faire en s'appuyant sur lui ${ }^{4}$.

(1b) Leukozyten sind farblose, kernhaltige Blutzellen. Sie haben keine feste Form. 
$\rightarrow$ Les leucocytes sont des cellules sanguines incolores à noyau. Ils n'ont pas de forme définie.

7 Les opérations de reformulation qui nous intéressent dans cette étude présentent deux configurations possibles : le vulgarisateur peut tout d'abord procéder à une définition. Dans ce cas, il peut choisir d'opérer par une définition terminologique « classique " où le terme est reformulé à l'aide de syntagmes nominaux, verbaux, etc., ainsi qu'en témoigne l'exemple (3) :

(3) Der Gasaustausch, d.h. die Abgabe von Kohlendioxid und die Aufnahme von Sauerstoff, findet in den Lungenbläschen statt, deren Gesamtoberfläche fast hundert Quadratmeter beträgt.(Atlas der Anatomie, 152)

$\rightarrow$ L'échange gazeux, c'est-à-dire le rejet de dioxyde de carbone et la prise/ absorption d'oxygène, a lieu dans les alvéoles pulmonaires, dont la surface totale atteint presque $100 \mathrm{~m}^{2}$.

8 Mais le vulgarisateur peut également opérer par ce que nous avons appelé une «définition intercodique par synonymie ou équivalence », dont l'exemple (1) mentionné ci-dessus est une illustration. Le segment source contient le terme («Leukozyten»), le segment reformulateur renferme le mot du code courant correspondant («weiße Blutkörperchen »). Ces reformulations se distinguent par le fait que, contrairement à une définition "classique ", c'est le passage éclair d'une unité minimale à une autre unité minimale entre deux codes différents, qui doit favoriser la compréhension et la réception par le lecteur. Ce type de reformulation est très fréquent en vulgarisation médicale allemande, car le même objet du monde peut être désigné soit par un terme, soit par un mot du code courant, contrairement au français pour lequel ce phénomène se cantonne à quelques exemples.

9 En mouvement inverse, le vulgarisateur peut opérer par dénominations, soit de manière classique par dénomination (voir exemple 4), soit par "dénomination intercodique par synonymie ou équivalence », illustrée par l'exemple (5) qui renferme trois dénominations intercodiques par synonymie ou équivalence :

(4) Unter Plasma versteht man den Anteil des Blutes ohne Zellen. Das Blutplasma ist eine klare, gelbliche, eiweiß- und salzhaltige Flüssigkeit. Die Farbe Rot entsteht übrigens durch den in den Erythrozyten enthaltenen roten Blutfarbstoff, das Hämoglobin.(Der Mensch, 59)

$\rightarrow$ Par plasma, on entend la partie du sang sans cellules. La plasma sanguin est un liquide clair, jaunâtre, salin et contenant des protéines. La couleur rouge naît, du reste, du pigment (sanguin) rouge contenu dans les érythrocytes, l'hémoglobine.

(5) Blut besteht im Wesentlichen aus zwei Grundbestandteilen, den Zellen und dem Plasma. Die Zellen sind dieroten Blutkörperchen (Erythrozyten), die weißen Blutkörperchen (Leukozyten) und die Blutplättchen (Thrombozyten). (Der Mensch, 59)

$\rightarrow$ Le sang se compose pour l'essentiel de deux composants de base, les cellules et le plasma. Les cellules sont les globules rouges (érythrocytes), les globules blancs (érythrocytes) et les plaquettes (thrombocytes).

Dans tous les cas, le locuteur opère un changement de code ou « code-switching " 5

Ces deux mouvements, définitions et dénominations, montrent que chaque code a pour fonction d'être "métacode » de l'autre. "C'est [...] tantôt le mot scientifique qui est désigné comme corps étranger relativement à la "langue" supposée du récepteur, tantôt, à l'inverse, les mots du code courant qui suscitent une prise de distance par rapport à la "langue" scientifique ». (J. Authier, 1982 : 42). De manière générale, quel que soit le public et quel que soit le vulgarisateur, on remarque toutefois que, dans les textes de vulgarisation, les termes sont livrés au lecteur essentiellement par des 
dénominations et non par des définitions (1/3 contre $1 / 4)$. Cela signifie que les ouvrages de vulgarisation tendent davantage à présenter un terme nouveau, en le mettant en exergue afin de le souligner comme étranger, qu'à l'intégrer comme faisant partie du vocabulaire courant.

Le discours de vulgarisation, selon certains auteurs ayant travaillé le sujet, se marquerait par le fait qu'il vise à une certaine intégration ; c'est-à-dire que après avoir été expliqué, précisé, dénommé, le terme serait intégré par le discours et serait repris ensuite sans reformulation, passant ainsi à l'intérieur du discours et n'étant plus stigmatisé comme "venant d'un Autre ». Le texte passerait donc à un autre niveau en permettant, par un effacement progressif des suites, l'appropriation des termes par le lecteur. L'étude de nos exemples a cependant infléchi partiellement ces affirmations. Si cela est vrai au niveau micro, sur une progression thématique de quelques unités, ceci ne s'inscrit toutefois pas dans un développement textuel continu d'une grande ampleur. En effet, les mêmes termes ou combinaisons lexicales spécialisés sont en permanence redéfinis ou renommés au fil des pages. Cette répétition doit tout d'abord être vue comme une stratégie rédactionnelle, dans le sens où la définition (par exemple) d'un terme n'est jamais considérée par le vulgarisateur comme acquise pour le lecteur. D'autre part, la répétition des mêmes reformulations (sous des formes chaque fois légèrement différentes) est liée à la structure même des textes de vulgarisation : ceux-ci sont écrits dans le but de pouvoir être consultés dans un ordre aléatoire en fonction du sujet intéressant le lecteur. Ils ne s'inscrivent donc pas dans un processus de lecture suivie comme le fait le lecteur pour un roman. Dans cet esprit rédactionnel, le fait que les définitions et dénominations des termes réapparaissent sans cesse relève d'une nécessité absolue.

\section{Les émissions télévisées de vulgarisation ${ }^{6}$}

La part occupée par les termes dans les émissions de vulgarisation est bien moins importante que dans les ouvrages de vulgarisation. L'image est un élément majeur d'explication de ce phénomène. Si celle-ci est un outil venant appuyer ou compléter les définitions ou dénominations dans un ouvrage écrit (et vice-versa), elle apparaît, au niveau de l'audiovisuel, comme un élément prépondérant et dominant qui ne laisse pas aux éléments verbaux annexes la place d'exister. En effet, le fait de filmer l'objet du monde ou le procès en cours offre au téléspectateur une visualisation immédiate de l'objet du discours, sans que celui-ci ait besoin d'opérations de définitions, de concrétisations, etc., venant l'étayer.

À l'intérieur des émissions télévisées, l'utilisation des termes est très diverse et cette diversité est fortement liée à la nature du discours, à la personne vulgarisant et aux buts poursuivis par celle-ci. Lorsque le vulgarisateur est un spécialiste que l'on interviewe, on constate que celui-ci s'applique à définir les termes qu'il emploie. En revanche, lorsque le vulgarisateur est un journaliste, la présence de reformulations définitoires ou dénominatrices est plutôt rare. Nous analyserons donc, dans un premier temps, les reformulations produites par des journalistes en tentant d'expliquer le faible taux de reformulations, puis nous nous intéresserons aux reformulations produites par des spécialistes interviewés. 


\section{Émissions télévisées et « journaliste-vulgarisateur »}

\section{Type de discours et présence restreinte de reformulations terminologiques}

15 Le discours du «journaliste-vulgarisateur» est un discours écrit, lu à haute voix. Il s'agit donc d'un discours préparé, rédigé, qui déroule un fil narratif, descriptif, voire argumentatif pensé en progression, duquel redondance et retour correctif (par exemple) sont exclus. Cela signifie que le terme apparaît une fois (soit en dénomination, soit en définition) à un moment précis car répondant à un besoin communicationnel, mais qu'il ne fait pas l'objet d'une deuxième mention pour une éventuelle redéfinition, par exemple. Le nombre très réduit de reformulations définitoires ou dénominatives dans le discours du "journaliste-vulgarisateur " s'explique donc en partie par le type de discours utilisé. Le deuxième élément d'explication de ce faible taux est lié aux objectifs de l'émission que le «journalistevulgarisateur " se doit de respecter et qui sont radicalement différents de ceux des ouvrages de vulgarisation. Alors que l'ouvrage de vulgarisation vise (entre autres) à apporter au lecteur les mots techniques et/ou à les définir, l'émission de vulgarisation vise plutôt, pour sa part, à informer sur les phénomènes. C'est ainsi qu'au lieu de s'interroger sur les protéines et leur définition, l'émission de vulgarisation mettra l'accent sur les endroits où l'on trouve les protéines, à leur utilité ou à l'usage que l'on en fait. L'important est que le téléspectateur retienne que c'est de protéines qu'il s'agit, sans se soucier de savoir exactement ce dont il s'agit. C'est ainsi que le journaliste utilise des termes qu'il sait être obscurs pour le téléspectateur, mais qui, malgré tout, ne feront pas l'objet d'une définition ou explication, ainsi que le montre l'exemple (6) dans lequel ni " protéines », ni " acides aminés » ne sont expliqués. ${ }^{7}$

(6) Sie ${ }^{8}$ unterscheiden sich kaum von werkstoffen wie polyester/die er normalerweise erforscht \schuld am schaum von Elklar/sind proteine/ die beim aufschlagen/aminosäureknäuel entfalten und so zur stabilisIErung der schaumstruktur beitragen\an die grenzschicht zwischen luftblase und eiklar setzen sich lange fäden-- die den schaum in form halten $\backslash(\mathrm{Nano}, 16 / 01 / 2006)$

$\rightarrow$ Elles se différencient peu des matériaux comme le polyester qu'il étudie normalement. Les responsables de la mousse du blanc d'œuf battu sont les protéines qui, lorsqu'on les bat, développent des pelotes d'acides aminés et qui contribuent ainsi à la stabilisation de la structure de la mousse. Sur la couche frontière entre bulle d'air et blanc d'œuf se déposent de longs fils qui font tenir la mousse.

16 Au-delà de ce simple constat, il est important de souligner que cette volonté de privilégier une information qui vise un contenu, plus qu'une information qui vise la terminologie, pose un problème de fidélité et d'exactitude scientifique. En effet, dans l'exemple (7), la reformulation proposée par le journaliste met sur le même pied d'égalité l'air expiré et le dioxyde de carbone :

(7) die langsame beatmung hat den vorteil/dass die VERBRAUCHTE atemluft/* also das KOHLENdioxid $\backslash$ gut abtransportiert wird.(Nano, 10/01/2006)

$\rightarrow$ La respiration (artificielle) lente présente l'avantage que l'air expiré (= vicié), c'est-

à-dire le dioxyde de carbone, soit bien transporté.

17 Les deux seraient donc identiques référentiellement. Or, le texte résumé de l'émission proposé sur Internet est le suivant :

(8) ...,dass das Kohlendioxid in der verbrauchten Atemluftgut abtransportiert wird. ${ }^{9}$

$\rightarrow \ldots$, que le dioxyde de carbone dans l'air expiré (= vicié) soit bien transporté. 
C'est uniquement grâce à la version écrite que le téléspectateur (curieux et zélé !) découvre que les deux ne sont pas identiques référentiellement, mais que l'un est en réalité un composant de l'autre. Cela signifie que l'émission télévisée vise une information facile pour laquelle on simplifie à l'aide de raccourcis, et qui amène à créer des équivalences qui n'en sont pas... Ce mode opératoire pose un problème au niveau scientifique, puisque ce type d'information se fait au détriment de l'exactitude scientifique (cet exemple est loin d'être une exception), et il pose également un problème éthique au niveau de la vulgarisation, puisque l'on peut dès lors s'interroger sur la nature et la valeur de l'information transmise et, par là même, sur le respect du public cible.

19 Les quelques rares opérations sur des termes auxquelles procèdent les journalistes vulgarisateurs sont essentiellement les dénominations, les réductions ou introductions de sigles et les définitions.

\section{Dénominations}

L'opération de dénomination sur un terme scientifique apparaît dans ce que l'on pourrait appeler une situation "sans issue» pour le "journaliste-vulgarisateur». Situation « sans issue » signifie que le journaliste est contraint d'utiliser en permanence le terme scientifique $\Omega$, dans la mesure où celui-ci est le terme usité, unique, dont le journaliste a besoin et sans lequel il ne peut rien faire. Il lui faut donc présenter cet $\Omega$, le porter à la connaissance du téléspectateur, l'amener dans le discours. Pour ce faire, le journaliste exprime dans le dit la nature de ce dont il va parler et fournit dans le redit le terme scientifique dont il se servira exclusivement par la suite, ainsi que le montre l'exemple (9) dans lequel «ein bislang vernachlässigter blutwert» permet d'introduire « $\gamma \mathrm{GT} »$ :

(9) das gilt insbesondere für herzerkrankungen/immer noch eine haupttodesursache| wissenschaftler aus innsbruck/haben eine sensationelle entdeckung gemacht -- ein bislang vernachlässigter blutwert $* / / \gamma G T / /$ soll aufschluss geben über das risiko an einer herzerkrankung zu sterben/oder einen schlaganfall zu erleiden $\backslash$ (Nano, 12/01/06)

$\rightarrow$ Cela vaut en particulier pour les maladies cardiaques, toujours à l'heure actuelle une cause principale de mortalité. Des scientifiques de Innsbruck ont fait une découverte sensationnelle : un hémogramme négligé jusqu'à présent,le $\gamma \mathrm{GT}$,informerait sur le risque de mourir d'une maladie cardiaque ou d'être touché par une attaque.

Pour introduire ces dénominations, le journaliste n'utilise pas de marqueurs lexicaux ${ }^{10}$. Il se contente toujours de faire une pause avant le redit, le dit étant, pour sa part, formulé sur un ton très montant (voir exemple 9 ci-dessus). Cet usage rappelle ainsi les emplois propres aux textes de vulgarisation où la dénomination (concernant le terme scientifique) est introduite par des marqueurs typographiques «silencieux»: parenthèses, tirets ou virgules.

À ces pauses et accentuations montantes s'ajoute parfois l'adjectif sogenannt-, présent dans le segment reformulateur contenant le terme, comme le montre l'exemple (10):

(10) So sind eileiter/gebärmutter/und der obere anteil der scheide zunächst gemeinsames gewebe/und teilen sich dann in der achten embryonalen woche auf nach einer komplizierten wanderung/nehmen sie ihre endgültige position im becken ein $\mid$ gesteuert wird diese wanderung von * bindegewebszellen - so genannten * PLATZanweisern. (Nano, 09/01/06)

$\rightarrow$ Les trompes, l'utérus et le haut du vagin sont donc tout d'abord un tissu commun et ils se séparent ensuite dans la huitième semaine embryonale. Après une 
migration compliquée, ils prennent leur place définitive dans le bassin. Cette migration est pilotée par des cellules du tissu conjonctif, par ce qu'on appelle des ouvreuses. ${ }^{11}$

\section{l'opération de dénomination n'est pas la seule raison de l'emploi de «sogenannt-». En} liaison avec l'«autorité dénommante» précédemment évoquée, il convient de souligner que le terme du segment reformulateur est montré en tant que signe, c'est-àdire en fonction autonyme. Or, dans ces reformulations sans marques lexicales, le caractère autonymique d'un constituant n'est pas donné explicitement. "Sogenannt- » va permettre, dans un mouvement progressif, de reconnaître comme «relation métalinguistique et opacifiante » (J. Authier-Revuz, 1995 : 126) la relation établie entre les deux éléments que sont le dit et le redit, et donc de souligner le terme du redit comme en mention. Dans l'exemple (10), « sogenannt- " permet à Platzanweisern d'être affiché en tant que signe, uniquement en mention, de se présenter comme le dire d'un Autre, spécialiste.

"Sogenannt-" ne se limite toutefois pas à accompagner un autonyme. En effet, si Bindegewebszellen dans l'exemple (10) est en usage, réfère à un objet du monde, il est en même temps celui qui a provoqué l'emploi du terme autonymique Platzanweisern. Par le retour réflexif lié à l'opération de reformulation - dans un mouvement régressif donc et par l'autonyme précédé de sogenannt-, il devient le signe auquel est proposé un signe équivalent. Il se trouve donc, lui aussi, "opacifié ", en mention par rapport à la suite reformulatrice, et il apparaît que le terme introduit par la dénomination peut modaliser autonymiquement le nom ou expression du code courant contenu dans le dit.

\section{Sigles}

La présence de sigles dans le discours du « journaliste-vulgarisateur » relève du même esprit de situation d'indigence que celle évoquée ci-dessus pour la dénomination. Certains sigles fonctionnent comme de véritables termes; on peut citer à titre d'exemple la péridurale, exprimée par le sigle «PDA» en allemand. Celui-ci est employé aussi bien par le spécialiste que par le profane comme terme scientifique, et bien peu d'Allemands connaissent son développement (Periduralanästhesie). Ces sigles étant incontournables, le journaliste va, à l'aide de reformulations, les introduire dans son discours selon le même procédé que la dénomination (souvent par réduction), de manière à les utiliser par la suite sans entrave. Contrairement aux dénominations, toutefois, les sigles sont introduits par le marqueur kurz (traduit ici par « abrégé en ») si le journaliste opère par réduction, comme dans l'exemple (11) ${ }^{12}$ :

(11) Innsbrucker forscher haben nun einen vollkommen neuen risikofaktor für herzkreislauferkrankungen gefunden\gammaglytamyltransferase \ $\underline{\mathrm{kurz}}^{*} \boldsymbol{\gamma} \mathrm{GT}$, ist ein leberenzym/dessen wert einfach bei der bluteinnahme festgestellt werden kann\(Nano, 12/01/06)

$\rightarrow$ Des chercheurs de Innsbruck ont trouvé un facteur de risque complètement nouveau pour les maladies cardio-vasculaires. La Gamma Glutamyl Transpeptidase, 
abrégée en $\mathbf{V G T}$, est une enzyme du foie, dont les valeurs peuvent être constatées par une simple prise de sang.

\section{Définitions}

Les définitions produites par le «journaliste-vulgarisateur» se distinguent par la présence de marques de reformulation lexicales ${ }^{13}$, dont la plus fréquente est $" N$ ist » (« $\mathrm{N}$ est », où $\mathrm{N}$ est une variable, anaphorique d'un élément du segment source ${ }^{14}$ ). Deux données rédactionnelles méritent d'être soulignées quant à l'emploi de cette marque de reformulation et des définitions qu'elle introduit: on peut tout d'abord remarquer que ces reformulations définitoires sont produites à un moment très particulier et bien délimitable, à savoir dans une partie que nous appellerions « introductive » où le journaliste est amené à présenter le thème du jour. Ce thème étant désigné par le terme qu'il va devoir utiliser, le journaliste définit celui-ci une fois pour toutes, afin de pouvoir s'en servir par la suite (exemple 13). Ce mode de fonctionnement n'est pas sans rappeler certaines dénominations évoquées ci-dessus. La différence réside cependant dans le fait que la situation «sans issue " décrite précédemment pour les dénominations n'est pas fixée dans un espace-temps, contrairement au cas présent.

(13) Generell ist diese behandlung durchgeführt worden/bei Patienten mit so genannten* autoimmunerkrankungen ${ }^{*}$ das sind erKRANKungen, wo sich das abwehrsystem GEGENden eigenen körper richtet.(18/01/2006)

$\rightarrow$ De manière générale, ce traitement a été mené chez des patients souffrant de ce qu'on appelle des maladies auto-immunes, ce sont des maladies, dans lesquelles le système immunitaire se dirige contre son propre organisme.

Le second point qui mérite attention est le fait que cette marque « $N$ ist » est fréquemment employée dans les émissions de vulgarisation quel que soit le public cible visé, alors qu'elle est quasiment absente des ouvrages de vulgarisation pour adultes profanes, son utilisation étant réservée au public enfantin. À une marque très explicite répondent dans ces ouvrages, des marques ambiguës (typographiques) qui réclament toutes une interprétation quant à la nature de l'opération effectuée, ou quant à la nature de la reformulation produite.

\section{Émissions télévisées et spécialiste vulgarisateur interviewé}

Si le discours du «journaliste-vulgarisateur » se marque par de rares retours sur les termes employés, le discours du spécialiste vulgarisateur interviewé, en revanche, est caractérisé par un nombre foisonnant de reformulations terminologiques, si bien que l'on pourrait parler d'emplois quasi-systématiques.

L'explication majeure de cette attention pour les termes réside dans le caractère dialogique du discours produit (dialogique au sens de M. Bakhtine (1929, trad. 1977) ${ }^{15}$. Dans la situation d'interview, la prise en considération par le spécialiste vulgarisateur de l'interlocuteur - fut-il le journaliste intervieweur ou le téléspectateur non physiquement présent - est permanente et considérable; soucieux de respecter le principe de coopération et en particulier, les maximes de quantité et de modalité (H.P. Grice, 1979), le spécialiste interviewé, conscient des difficultés que représente un terme pour un non-spécialiste, éprouve constamment le désir de bien se faire comprendre par son interlocuteur (intervieweur et téléspectateur) et fait ainsi preuve de véritables efforts lors de sa production orale. Se rendant compte que le terme scientifique ou 
technique peut être assimilé par le profane à du jargon, il revient sur son dit pour le clarifier, étant amené ainsi à se reformuler sans cesse. La reformulation permet par exemple, en présentant le même référent sous une forme différente, de respecter la maxime de modalité, si la première formulation contenant le terme s'avère obscure pour le téléspectateur. Elle peut également permettre de respecter la maxime de quantité si ce même premier énoncé ne contient pas assez d'informations, puisque le terme ne «dit» rien au profane. Enfin, lors de tous ses retours sur ses dits, le spécialiste vulgarisateur interviewé vise au respect de la maxime de qualité, puisque dans ses définitions, explications, etc. du terme scientifique ou technique, il vise à transmettre des informations et des connaissances exactes à un téléspectateur néophyte qu'il s'agit de ne pas induire en erreur. Même si une reformulation à l'identique est très difficile, puisque la reformulation altère un dit "autrement équivalent» (J.Peytard, 1984: 17-18, 1994 et 2000), et puisque, ainsi que le fait remarquer C. Norén (1998 et 1999), les contours du référent sont toujours un peu flous, les contenus des deux segments ne se recoupant jamais tout à fait et des nuances, des détails ou même des informations se perdant lors du passage du terme au mot du code courant (et vice-versa), le spécialiste vulgarisateur interviewé s'efforce en permanence de fournir un dit ou un redit aussi fidèle que possible par rapport à un terme supposé problématique. En ce sens, son comportement (exemplaire!) peut être considéré comme le pendant de celui du «journaliste-vulgarisateur » évoqué précédemment, puisque son comportement vise à privilégier à la fois une information qui vise un contenu et une information qui vise la terminologie. ${ }^{16}$

31 Comme dans les textes de vulgarisation, le spécialiste interviewé produit soit des "définitions intercodiques par synonymie ou équivalence", soit des définitions terminologiques dont (14) est un exemple :

(14) Man wollte zunächst herausfinden/mit welchen chemischen stoffen die glasur am wirksamsten haften bleibt \wir haben verschiedene Tests durchgeführt \am besten dabei abgeschnitten// hat polyvinyl butyral $\backslash$ POLYVINYL BUTYRAL// ist ein synTHETIsches harz। und es weist für unsere ${ }^{* *}$ äh ${ }^{* *}$ zwecke die besten festigungseigenschaften auf $\mid$ (Nano, 09/01/06)

$\rightarrow$ On voulait tout d'abord découvrir avec quelles substances chimiques la glaçure tiendrait le mieux. Nous avons effectué différents tests. Ce qui a marché le mieux a été le polyvinyle de butyral. Le polyvinyle de butyral est une résine synthétique et il présente pour nos... euh... buts les meilleures propriétés de consolidation.

Le spécialiste vulgarisateur interviewé peut également produire ce que nous appelons des "reformulations concrétisantes". La perspective énonciative est alors de réexprimer le contenu du dit de manière plus concrète. Le redit permet de situer la signification du dit au niveau des faits, en ancrant un contenu souvent vague, trop abstrait ou théorique dans la réalité pour en montrer la correspondance «sur le terrain ». C'est le cas de l'exemple (15) dans lequel le chimiste précise ce que veut dire "dénaturer mécaniquement " en expliquant concrètement ce qui se passe au niveau des protéines :

(15) Wenn sie jetzt mit dem mixer reingehen/dann bekommen sie zwei effekte $\backslash$ zum einen/ schlagen sie luft ein \dann bilden sich diese bläschen/und zum zweiten/wird eben dieses eiweiß sozusagen mechanisch denaturiert/das heißt ${ }^{*}$ diese knäuel/diese dichten packen knäuel/die entfalten sich zu langen fäden \(Nano, 16/01/06)

$\rightarrow$ Lorsque maintenant vous rentrez dedans avec le mixer, vous obtenez deux effets : premièrement vous faites rentrer de l'air et ces petites bulles se forment alors, et deuxièmement ce blanc d'œuf est pour ainsi dire dénaturé mécaniquement, 
c'est-à-dire que ces pelotes, ces piles épaisses de pelotes, elles se déploient en de longs fils. d'hypertexte et celle-ci est à la base même de traitements particuliers concernant termes scientifiques. À la suite de A. Storrer ${ }^{18}$, nous dirons qu'un hypertexte est un texte organisé de manière non linéaire et géré par une technique informatique ${ }^{19}$. À cette définition générale viennent s'ajouter au moins quatre propriétés: le codage multimodal (données textuelles, picturales, audio et vidéo), la dynamique (articles pouvant être ajoutés à l'article initial, le contenu de celui-ci modifié, etc.), l'interactivité (interaction entre l'utilisateur et le système informatique) et la communication au moyen d'ordinateurs (interaction des utilisateurs entre eux) (A. Storrer, 2008). Notons que le critère de dynamique se trouve quelque peu restreint dans les sites de vulgarisation médicale que nous avons étudiés, puisqu'ils se composent essentiellement d'hypertextes dont le contenu n'est pas modifié par les utilisateurs, la rédaction en revenant exclusivement à un journaliste dit «spécialisé » ou à un professionnel de la santé20. En lien avec le codage multimodal et le critère d'interactivité, il convient de distinguer entre l'hypertexte et ce que A. Storrer appelle le «e-texte ». Ce dernier est organisé de manière linéaire, avec un début et une fin, il est pensé pour être lu dans son ensemble et ne comporte pas de liens sur lesquels l'utilisateur peut cliquer. Les articles scientifiques en PDF, les œuvres littéraires digitalisées en sont des exemples. A contrario, un hypertexte se marque par une grande présence de liens (internes à un paragraphe ou extérieurs) menant le lecteur vers d'autres pages. On constate que les sites de vulgarisation français et allemands ont opté pour un mélange des genres : si ceux-ci reposent essentiellement sur des hypertextes composés de divers liens, images, mini-résumés, etc., on remarque que certains liens peuvent mener l'utilisateur vers un texte qui s'apparenterait à un e-texte dénué de tout lien électronique, relevant donc davantage du texte figé dans une forme fermée sur elle-même. Ces deux «types de textes » ont des conséquences importantes sur le mode de vulgarisation. En effet, si l'attention portée aux termes est la même sur ces sites spécialisés que celle propre aux textes de vulgarisation, le traitement des termes, pour sa part, diffère en raison de la configuration électronique.

Les mêmes types de reformulations intratextuelles que ceux que nous avons présentés au début de cet article à propos des textes de vulgarisation sont utilisés, en particulier 
les reformulations mettant en scène des définitions ou dénominations par synonymie ou équivalence, marquées par des parenthèses (qu'il s'agisse d'hypertextes ou de etextes). L'exemple (16) est une illustration de définition par synonymie ou équivalence :

(16) Eine Blutvergiftung ("Sepsis") ist eine schwere Allgemeininfektion, die durch Mikroorganismen, meist Bakterien, ausgelöst wird.

(http://www.surfmed.de/?news/df44001e184e2a81bd2d364c45082ca9)

$\rightarrow$ Un empoisonnement sanguin (« septicémie ») est une infection générale grave, qui est provoquée par des microorganismes, pour la plupart des bactéries.

Cependant, malgré ces ressemblances, le nombre de reformulations intratextuelles sous la forme $x R y$ (avec $\mathrm{X}$ et $\mathrm{Y}$ intégrés, comme syntagmes verbaux ou syntagmes nominaux constituants de syntagmes verbaux) est nettement moindre que dans les textes de vulgarisation. Ceci s'explique par deux facteurs :

La première raison est en lien avec une présentation qui s'apparente à celle d'un dictionnaire: le terme peut être isolé, donné comme un titre, tel une entrée de dictionnaire. Il peut également apparaître sous forme de question «Was ist $X$ ?» (« qu'est-ce que $X$ ? »). Dans les deux cas, s'ensuit une définition dont l'entrée en matière est souvent la même : "X ist... » ("X est... »), « bei X handelt es sich um... » (" il s'agit de... ») ou "unter $X$ versteht man...» («sous $X$ on comprend... ») ${ }^{21}$. Ainsi, dans l'exemple (17), «Diabetes mellitus» est le titre. Vient ensuite le corps de l'article commençant par « Beim Diabetes mellitus handelt es sich um », suivi de la définition:

(17) Diabetes mellitus (Zuckerkrankheit) ${ }^{22}$

Beim Diabetes mellitus handelt es sich um eine chronische Stoffwechselerkrankung, die

durch einen erhöhten Blutzuckerspiegel gekennzeichnet ist.

(http://www.onmeda.de/krankheiten/diabetes_mellitus.html)

$\rightarrow$ Par diabète mellitus, on entend une maladie chronique touchant le métabolisme,

qui est caractérisée par une concentration élevée de glucose dans le sang.

Si l'anaphorisation est ici évidente, on ne peut en revanche parler de reformulation intratextuelle au sens propre, à savoir d'une opération discursive résultant d'un retour réflexif par rapport à un segment source premier et engendrant un segment reformulateur suppressible, donné « en plus ». Dans cette présentation particulière, la vulgarisation par Internet se distingue radicalement de l'ouvrage de vulgarisation dans lequel une telle configuration ne peut apparaître dans le corps du texte (voir à ce sujet note 25).

La deuxième raison du nombre moindre de reformulations intratextuelles est liée au terme lui-même : lorsque celui-ci n'a pas d'équivalent en code courant, ou lorsqu'il y a matière à plus de développement qu'une simple équivalence, l'internaute a la possibilité de cliquer sur le terme scientifique. Par un lien, le profane atteint un autre article à propos de ce même terme. Ainsi, dans l'exemple (18), le lecteur peut cliquer en (18A) sur «Schwangerschaftsdiabetes » et atteint une autre page web représentée par (18B) :

(18)

A. Es werden hauptsächlich zwei Diabetesformen unterschieden: Typ-1-Diabetes beginnt meist in der Jugend und entsteht durch die Zerstörung der Insulin produzierenden Zellen. Typ-2-Diabetes betrifft dagegen meist ältere Menschen. Er beginnt langsam und beruht auf einer zunehmenden Unempfindlichkeit der Zellen gegenüber dem Insulin. Daneben gibt es noch einige seltenere Diabetes-Sonderformen und den Schwangerschaftsdiabetes.

$\rightarrow[. .$.$] À côté de ceci, il existe également quelques formes particulières de diabète$ plus rares et le «diabète de la femme enceinte »

B.Schwangerschaftsdiabetes(Stand: 11. Januar 2008)Unter Schwangerschaftsdiabetes (Gestationsdiabetes) versteht man einen erhöhten Blutzuckerspiegel der Mutter während der 
Schwangerschaft. In den Industrienationen kommt es bei fast fünf Prozent aller Schwangerschaften zu Schwangerschaftsdiabetes. Er äußert sich etwa ab dem siebten Schwangerschaftsmonat. Nach der Geburt verschwindet der Schwangerschaftsdiabetes von selbst; in äußerst seltenen Fällen handelt es sich auch um einen unerkannten Diabetes mellitus.

$\rightarrow$ «Diabète de la femme enceinte »

(Etat : 11 janvier 2008)

Par «diabète de la femme enceinte " (diabète gestationnel), on comprend une concentration élevée de glucose dans le sang de la mère pendant la grossesse. [...]

41 Le lecteur est donc invité à passer de pages web en pages web à l'intérieur de ce que E. U. Grosse et E. Seibold (1996) - et à leur suite J.-M. Adam et G. Lugrin (2000 et 2006, et G. Lugrin 2000 et 2001) - ont appelé dans le cadre de la presse quotidienne une «hyperstructure $»^{23}$. En liaison avec celle-ci, la notion de " parcours de lecture $»^{24}$ est ici intéressante. Ces liens hypertextes hiérarchisés amènent à sortir du cadre de l'intratextuel stricto sensu, et ceci a pour conséquence de bloquer toute reformulation intratextuelle à proprement parler, dans la mesure où le segment reformulateur ne peut suivre immédiatement dans la continuité phrastique le segment source. Le traitement du terme a donc lieu, dans ces configurations, hors du cadre de l'opération de reformulation ${ }^{25}$.

42 La deuxième conséquence découlant de l'interface particulière des liens menant vers des pages web est que le lecteur internaute ne peut cliquer que sur des termes. Cela signifie que le terme scientifique ne peut faire l'objet que de définitions, et les dénominations se trouvent donc exclues de ce procédé. En ce sens, le parcours de lecture multimédia limite le champ des possibles quant à la présentation du terme scientifique.

43 Au-delà de la question du traitement du terme, il convient de remarquer que la présence de ces liens hypertextes - reconnus comme très pratiques au demeurant pose toutefois un problème au niveau de l'entreprise de vulgarisation : en effet, si les sites Internet sont réalisés dans le même esprit que le texte de vulgarisation, c'est-àdire consultables eux aussi de manière aléatoire, la grande différence réside dans le fait que le cours de la lecture peut être entravé, dans la mesure où il est facile de se perdre au fil des liens proposés. Il y a donc là une véritable rupture dans la progression textuelle qui est très dommageable au niveau informationnel, et on peut se demander si la notion même de parcours (au sens de "parcours guidé ») est alors justifiée. Par ailleurs, on peut s'interroger également, dans ce cadre-là, sur les problèmes de frontières des unités, de cohérence et de cohésion textuelle dans cette hyperstructure multimédia. L'objet de cette contribution n'est pas de discuter le problème de cohérence et cohésion de l'hypertexte, la réponse à la question de savoir si un hypertexte est cohérent est très délicate et mérite plus de deux lignes ${ }^{26}$. Intéressonsnous cependant toutefois au problème de la cohérence du point de vue du codeur, c'està-dire ici du vulgarisateur. Du fait de la non linéarité de l'organisation textuelle et des divers chemins de lecture qui peuvent être empruntés, la réception du contenu est, au niveau de la succession des informations, différente chez chaque individu. Cela signifie que le vulgarisateur ne peut anticiper un cheminement unique, il lui est impossible de savoir de quelles informations le lecteur disposera et à quel moment, et tout ceci a des conséquences sur la planification de la cohérence. Le vulgarisateur doit en effet définir, dénommer, expliquer le terme dans différents modules à différents endroits, afin de pallier un éventuel manque de connaissances du lecteur. Ceci conduit à une certaine redondance dans le contenu, qui pourtant devra par ailleurs progresser. Cette dernière 
dimension pose tout le problème de l'intertextualité, des bornes d'un texte et de la définition même de celui-ci. Ainsi, par l'analyse des termes sur des sites de vulgarisation en ligne, c'est toute la notion de texte qui est à interroger, notamment dans le rapport intertextuel/intratextuel et hypertextuel/intertextuel.

\section{Conclusion}

Cette étude des différents médias a montré que les vulgarisateurs de textes de vulgarisation, de sites web et d'émissions télévisées, lorsque l'interviewé est spécialiste, procèdent de manière très similaire en plaçant le terme au cœur de leur discours et en lui portant toute leur attention. Face à eux, se trouve le journaliste d'émissions télévisées qui ne définit et ne dénomme le terme qu'en situation d'« indigence », c'està-dire s'il ne peut pas faire autrement, car il a besoin de celui-ci pour la suite de son discours. En ce sens, il apparaît que le terme, dans son traitement de vulgarisation, dépend plus du "Troisième Homme » que du média support. Nous avons montré, par ailleurs ${ }^{27}$, que la manière de vulgariser/reformuler les termes du Spécialiste est fortement liée aux publics-cibles. Tout est donc interaction, le terme étant la clé de voûte autour de laquelle s'articule la structure spécialiste-vulgarisateur-profanesupport, éléments indissociables, inamovibles et à l'origine même des tribulations du terme scientifique ou technique.

La question qu'il convient de se poser au regard de ces analyses est de savoir si, du point de vue du profane demandeur de savoir, les trois média étudiés se placent dans un rapport d'exclusion les uns par rapport aux autres et se suffisent à eux-mêmes, ou si, au contraire, ils se situent dans des rapports complémentaires, les uns offrant des possibilités non offertes par les autres. Une troisième proposition, plus pessimiste, voudrait voir une redondance inutile de tous ces supports. Nous sommes d'avis que ces questions ne peuvent trouver de réponses que par l'étude des publics-cibles, de leurs attentes et de leurs modes de fonctionnement face aux différents médias dans le cadre de la vulgarisation et hors du cadre de celle-ci.

\section{BIBLIOGRAPHIE}

\section{Émissions télévisées}

Nano (3Sat)

\section{Références bibliographiques}

Dobers J., Jaenicke J. \& Rabisch G., Erlebnis Biologie, Hannover, Schroedel Verlag, 256 p., 1999.

Brott, A. et al., Biologie 2/3, Berlin, Cornelsen Verlag, 312 p., 1995.

Söhl, C., Der Mensch, Nürnberg, Burgschmiet Verlag, 79 p., 2001.

Weitz, B. (Dr.), Atlas der Anatomie, KometMA-Service und Verlagsgesellschaft mbH, Frechen, 318 p., 2001. 


\section{Sites Web cités}

http://www.surfmed.de

http://www.onmeda.de

\section{Auteurs et ouvrages cités}

Adam J.-M. \& Lugrin, G., «L'hyperstructure : un mode privilégié de présentation des événements scientifiques ", in Fabienne Cusin-Berche (dir.) : Rencontres discursives entre science et politique. Spécificités linguistiques et constructions sémiotiques, Carnets du CEDISCOR, $\mathrm{n}^{\circ}$ 6, Presses de la Sorbonne Nouvelle, p. 133-149, 2000.

Adam J.-M. \& Lugrin, G., « Effacement énonciatif et diffraction co-textuelle de la prise en charge des énoncés dans les hyperstructures journalistiques ", Semen, 22, Énonciation et responsabilité dans les médias. [En ligne], 2006.

Antos, G.,Grundlagen einer Theorie des Formulierens. Textherstellung in geschriebener und gesprochener Sprache, Tübingen, Gunter Narr, 1982.

Authier-Revuz, J., " La mise en scène de la communication dans des discours de vulgarisation scientifique ", in Langue Française ${ }^{\circ}$ 53, p. 34-47, 1982.

Authier-Revuz, J., Ces mots qui ne vont pas de soi. Boucles réflexives et non-coïncidences du dire, 2 Tomes, Sciences du Langage, Larousse, Paris, 1995.

Bakhtine, M., Le marxisme et la philosophie du langage, Paris, Les Editions de Minuit, 1929, trad. 1977.

Bakhtine, M., Esthétique de la création verbale, Paris, Gallimard, 1979, trad. 1984.

Grice H.P., « Logique et conversation » in Communications, $\mathrm{n}^{\circ}$ 30, p. 57-72, 1979.

Grosse E.U. \& Seibold E., « Typologie des genres journalistiques », in Grosse E.U. \& Seibold E. (éd.) : Panorama de la presse parisienne, Berne-Berlin, Peter Lang, p. 32-60, 1996.

Gumperz, J. J., Sociolinguistique interactionnelle. Une approche interprétative, Paris, L'Harmattan, 1982, trad. 1989.

Lerat, P., Les langues spécialisées, Paris, P.U.F., 1995.

L'Homme, M.-C., « Définition du statut du verbe en langue de spécialité et sa description lexicographique », in Cahiers de lexicologie 73 (2), p. 61-84, 1998.

L'Homme, M.-C. \& Vandaele, S., (éd). Lexicographie et terminologie : compatibilité des modèles et des techniques, Ottawa, Les Presses de l'Université d'Ottawa, 2007.

Lugrin, G., « Les ensembles rédactionnels : multitexte et hyperstructure », Médiatiques, Louvainla-Neuve, Observatoire du récit médiatique, p. 34-36, 2000.

Lugrin, G., « Le mélange des genres dans l'hyperstructure », Semen, 13, Genres de la presse écrite et analyse de discours. [En ligne], 2001.

Moirand, S., « De l'aire de la page à l'hyperstructure et à l'écran : comment lire et analyser la presse quotidienne ordinaire », in Cauce, Revista Internacional de Filologia y su Didàctica $n^{\circ} 29$, p. 295-320, 2006.

Norèn, C., «Reformulation et échelles argumentatives ", in Actes du XIIIe congrès des romanistes scandinaves, Jyväskylä, p. 441-454, 1998.

Norèn, C., Reformulation et Conversation, de la sémantique du topos aux fonctions interactionnelles, Uppsala, Acta Universitatis Upsaliensis, 1999. 
Peytard, J., « Problématique de l'altération des discours : reformulation et transcodage », in Langue Française, $\mathrm{n}^{\circ}$ 64, p. 17-28, 1984.

Peytard, J., « De l'altération et de l'évaluation des discours », in Moirand, S., Bouacha, A.A., Beacco, J.-C., Collinot, A. (éds), Parcours linguistiques de discours spécialisés, p. 69-80, 1994.

Peytard, J., « De l'altération discursive : regards sur le carrefour Mikhaill Bakthine/Gilles Deleuze », in Anderson, P., Chauvin-Vileno, A., Madini, M. (éds), Répétition, altération, reformulation, p. 23-36, 2000.

Rath, R., « Kommunikative Paraphrasen », in Linguistik und Didaktik, 6, p. 103-118, 1975.

Rath, R., Kommunikationspraxis, Analysen zur Textbildund und Textgliederung im gesprochenen Deutsch, Göttingen, Vandenhoeck und Ruprecht, 1979.

Storrer, A., « Was ist "hyper" am Hypertext ? ", in : Kallmeyer, Werner (Hg.) : Sprache und neue Medien. Berlin u.a. : de Gruyter (Jahrbuch 1999 des Instituts für deutsche Sprache), p. 222-249, 2000.

Storrer, A., « Kohärenz in Hypertexten », in : Zeitschrift für germanistische Linguistik 31.2., p. 274-292, 2004.

Storrer, A., « Hypertextlinguistik », in: Nina Janich (Hg.): Textlinguistik. 15 Einführungen. Tübingen, PDF-Preprint : [En ligne], 2008.

Vargas, E., Procédés de reformulation intratextuelle dans les ouvrages de vulgarisation en allemand. Etude d'une opération métalangagière et de ses marques, Thèse de doctorat, Paris IV Sorbonne, 1369 p., 2005.

Vargas, E., « La reformulation intratextuelle dans le texte de vulgarisation : un outil de didactisation du savoir ", in Pratiques sociales et didactiques des langues, Etudes offertes à Claude Vargas, PUP, p. 111-134, E. Vargas, V. Rey et A. Giacomi (éd.), 2007.

\section{ANNEXES}

\section{Conventions de transcription}

dioxid -- ton monocorde

Dioxid / ton montant

Dioxid // ton doublement montant

dioxid \ton descendant

dioxid $*$ pause courte

dioxid $* *$ pause longue

DIOxid accent très prononcé

\section{NOTES}

1. Voir M.C. L'Homme (1998), M.C. L'Homme \& S. Vandaele (2007).

2. Voir E. Vargas (2005).

3. Sur les reformulations non-paraphrastiques, voir E. Vargas (2005). 
4. La progression textuelle peut ainsi se réaliser par de véritables chaînes reformulatives. C'est ce qu'illustre l'exemple (2) ci-dessous, exemple d'une progression à thème linéaire, où chaque rhème d'un premier énoncé devient thème de l'énoncé suivant. Dans l'exemple ci-dessous, « einen hohen Anteil an Stärke und Zucker » constitue le rhème du premier énoncé. Une partie de ce rhème "Stärke und Zucker» est sélectionnée et devient élément thématique dans l'énoncé suivant. Le segment reformulateur («Kohlenhydrate») est rhématique. Celui-ci est à son tour thématisé dans l'énoncé suivant acceptant comme nouveau rhème «Kraftstoffe » :

(2) Backwaren, Nudeln, Reis, Kartoffeln und Süßigkeiten enthalten einen hohen Anteil an Stärke und Zucker. Stärke und Zucker sind Kohlenhydrate. Kohlenhydrate sind "Kraftstoffe". Sie haben Energie gespeichert.(Erlebnis Biologie : 148).

$\rightarrow$ Les viennoiseries, les pâtes, le riz, les pommes de terre et les sucreries renferment une forte proportion d'amidon et de sucre. L'amidon et le sucre sont des hydrates de carbone. Les hydrates de carbone sont des « carburants ». [...]

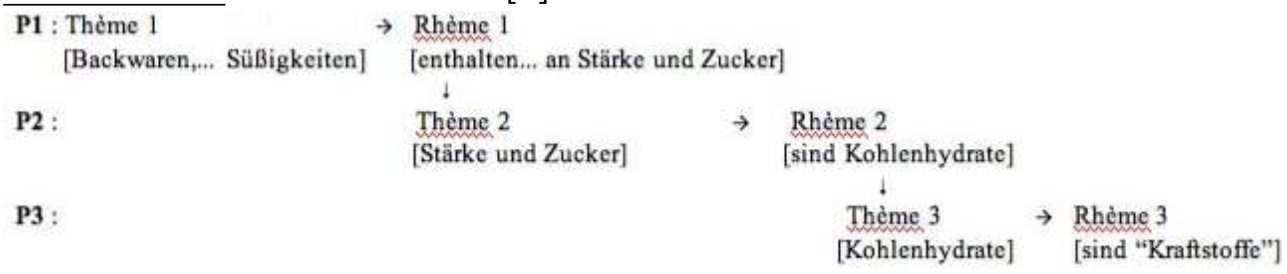

5. Le code-switching (ou "changement de code» ou "alternance codique ») peut se définir comme « [...] la juxtaposition à l'intérieur d'un même échange verbal de passages où le discours appartient à deux systèmes ou sous-systèmes grammaticaux différents. Le plus souvent l'alternance prend la forme de deux phrases qui se suivent. Comme lorsqu'un locuteur utilise une seconde langue soit pour réitérer son message soit pour répondre à l'affirmation de quelqu'un d'autre » (J. Gumperz, 1989, 57). Nous soulignons « soit pour réitérer son message », car cette indication concerne directement la reformulation. Cependant, nous avons été amenée à modifier en partie le concept de code-switching comme phénomène de changements de langue ou changement de registre, de variété (régionale, sociale, etc.) à l'intérieur d'une même langue. Notre corpus contient en effet, d'une part, des termes issus de ce que l'on appelle généralement les « langues spécialisées » (scientifique, technique) et des mots issus de la « langue commune ou courante » et, d'autre part, des mots issus de langues étrangères (anglais et espagnols). Dans un souci de clarté, le terme de « code » a été réservé pour désigner les variétés propres à la langue allemande et nous avons considéré l'activité de "code-switching» comme une activité désignant uniquement un changement de code (code courant, code spécialisé (scientifique ou technique) et code argotique), restreignant ainsi son acception générale, et l'appliquant à une situation monologale. Le terme de "langue " a été conservé pour désigner les langues étrangères. Nous distinguons donc entre traduction interlinguale et code-switching (intralingual). (Il est des cas où les deux se rencontrent. Nous ne pouvons pas les développer ici, nous renvoyons pour cela à notre travail de thèse. E. Vargas, 2005).

6. Diverses émissions ont été étudiées. Les exemples cités ici sont tous extraits de la même émission «Nano » diffusée par 3Sat. Cette émission quotidienne se définit comme une émission scientifique ayant pour but l'accessibilité de tous à la science, dans des domaines tels que la médecine, les sciences et techniques, les sciences humaines et naturelles, etc.

7. Nous retranscrivons les tonalités, pauses, etc. uniquement dans la version allemande des exemples, puisque d'une part celles-ci seraient différentes en français, et, d'autre part, car ces traductions n'ont jamais été prononcées.

8. Il s'agit des nanostructures des aliments mous.

9. Il est possible de cliquer sur dioxid pour en obtenir la définition.

10. Exception faite des sigles. 
11. La traduction en français de l'adjectif « sogenannt-» est délicate. On pourrait traduire ce terme par «ainsi appelé». Or, les formulations seraient assez malheureuses en français. « Sogenannt- » sert essentiellement à montrer qu'il s'agit d'un terme. Dans une traduction écrite, afin de pouvoir exprimer cette mise à distance entre les mots du code courant du lecteur et le terme, et afin de conserver le caractère autonyme du terme du segment reformulateur, il serait possible de traduire «sogenannt-» par des guillemets. Notre corpus de textes écrits atteste en effet que les guillemets ont les mêmes fonctions que « sogenannt- » pour ce qui est de l'autonyme et du soulignement d'un terme. Il s'agit ici d'un texte oral, les guillemets sont donc impropres, d'où notre choix de traduction par «ce qu'on appelle» qui ne nous satisfait toutefois pas complètement.

12. Le sigle est une alternative au terme en ce sens qu'il en présente une forme d'expression autre; malgré sa forme réduite, il convient donc de le considérer également comme terme. L'opération de reformulation qui les présente conjointement offre donc une configuration: Terme 1 (segment source) - Terme 1bis (segment reformulateur). (Sur les sigles, voir Vargas, 2005 et Lerat, 1995).

13. Le journaliste peut également opérer sans utiliser de marqueur lexical (voir exemple cidessous), mais cette tendance est assez réduite :

(12) Dann ein riechtest \die ärzte wollten wissen, ob und wie viel er riecht $\backslash$ EIner der düfte im test ist GERANIOL/ein bestandteil der rose \(Nano, 17/01/06).

$\rightarrow$ Ensuite un test d'odorat. Les médecins veulent savoir s'il sent et comment. Une des odeurs dans le test est le géraniol, un composant de la rose.

14. Sur ce type de définition où $\mathrm{N}$ est en général le terme hyperonyme, voir Vargas 2005.

15. À propos de la vulgarisation scientifique, M. Bakthine précise : «Par exemple, le genre de la vulgarisation scientifique s'adresse à un cercle précis de lecteurs, marqués par un certain fond aperceptif de compréhension responsive, c'est à un autre lecteur que s'adressent des écrits traitant de connaissances spécialisées, et c'est à un tout autre lecteur que s'adresseront les ouvrages portant sur des recherches spécialisées. Dans tous ces cas, il sera tenu compte du destinataire (et de son fond aperceptif) et l'influence de celui-ci sur la structure de l'énoncé est très simple : tout se ramène à l'ampleur relative de ses connaissances spécialisées » $(1984,304)$.

16. La notion même d'interview et de discours non préparé est évidemment également un élément explicatif de la présence de reformulations. Le caractère linéaire de la communication contraignant à ce que R. Rath appelle le "paraphrasage » (1975 et 1979) et la difficulté du processus de formulation selon la définition qu'en donne G. Antos (1982) sont les autres causes de la présence de reformulations.

17. Si ces notions sont intéressantes et essentielles pour décrire les mouvements observés, elles ne s'appliquent cependant pas aux reformulations s'effectuant hors code-switching. Il faudrait pour ces cas imaginer un mouvement fermé à l'extérieur et donc refermé sur lui-même, un mouvement en boucle.

18. Nous nous appuyons ici sur les travaux de A. Storrer $(2000,2004,2008)$, dans la mesure où ses recherches concernent essentiellement Internet. Nous renvoyons cependant également aux travaux sur l'hyperstructure de J.-M. Adam \& G. Lugrin $(2000,2006)$ et G. Lugrin $(2000,2001)$.

19. "Hypertexte sind nicht-linear organisierte Texte, die durch Computertechnik verwaltet werden " (Storrer, 2008).

20. L'identité du rédacteur n'est pas toujours facile à déterminer sur ces sites.

21. Ces présentations sont utilisées aussi bien dans les e-textes que dans les hypertextes.

22. On remarque une définition intercodique par synonymie ("Zuckerkrankheit») suivant le terme, mais qui n'est pas ici l'objet de notre propos.

23. "L'hyperstructure est un élément de structuration de l'information, intermédiaire et facultatif, situé entre le journal et l'article. Elle trouve son origine dans un processus d'éclatement ou de réunion. Elle est formée d'un ensemble d'articles et d'images graphiquement 
regroupés et complémentaires, bornés à la limite matérielle de l'aire scripturale visible de la double page " (G. Lugrin, 2001). Il est nécessaire, dans le cadre d'Internet, de revenir sur le dernier point : la limite matérielle ne peut se réduire à l'aire scripturale de la double page.

24. Voir entre autres G. Lugrin (2001) et S. Moirand (2006).

25. Une telle configuration avec «titre » et hors reformulation peut exister dans les ouvrages de vulgarisation, si ceux-ci comportent un glossaire. Toutefois, la consultation du glossaire ne relève pas d'un parcours de lecture guidé, puisque le terme est toujours défini ou dénommé dans le corps du texte. Le lecteur peut ainsi se passer du glossaire, et ce dernier n'est en fait qu'un outil supplémentaire pour qui veut ponctuellement une définition rapide d'un terme sans passer par le texte de vulgarisation lui-même.

26. Nous renvoyons pour cela aux travaux de A. Storrer (2003), en particulier à sa distinction entre cohérence locale et cohérence globale.

27. Voir Vargas $(2005,2007)$.

\section{RÉSUMÉS}

Cette contribution s'intéresse aux termes scientifiques médicaux et aux « combinaisons lexicales spécialisées » dans le cadre de la vulgarisation. La question à la base de ce travail est de savoir si ceux-ci subissent le même traitement au niveau de la vulgarisation dans les différents médias pouvant exister. Autrement dit: existe-t-il un type unique de vulgarisation ou faut-il, au contraire, parler de «modes de vulgarisation »? Cette étude s'est donc appuyée sur trois médias différents : des ouvrages de vulgarisation, des émissions de vulgarisation télévisées et des sites Internet de vulgarisation médicale spécialisés. Par ailleurs, les analyses effectuées ont pris en considération le vulgarisateur, ce Troisième Homme incontournable, afin de souligner l'importance ou la non-pertinence de celui-ci dans le traitement du terme. Ainsi, cet article se propose de montrer que le terme, lors du processus de vulgarisation, dépend souvent plus du «Troisième Homme » que du média support.

This paper studies the vulgarisation of scientific medical terms and "specialised lexical combinations", raising the question of whether such terms undergo the same vulgarisation processes according to the media concerned i.e., is vulgarisation a standardised process or are there different "modes of vulgarisation"? The study is based on three different kinds of media supports: vulgarised editions, popular television programmes and online sites specialising in vulgarisation of medical terms. It highlights the role of the indispensable "Third Person" i.e. the "vulgarisator", and probes the question of the degree of importance this "Third Person" represents in terms of the actual processing. To conclude, it proposes to show that vulgarisation processes depend more often on the "Third Person" than on a particular media.

\section{INDEX}

Mots-clés : vulgarisation, terme scientifique, médiatisation de la science, reformulation, hypertexte

Keywords : vulgarisation, scientific terms, mediatisation of scientific discourse, reformulation, hypertext 
AUTEUR

ELODIE VARGAS

Université Stendhal-Grenoble 3

ILCEA / GREMUTS 